\title{
A Method for Identifying Factual Corruption of the Navigation Signal and Identifying the Interference Influence on Unmanned Aerial Vehicle Receiver
}

\author{
Gennady Linets ${ }^{1}$ (D), Konstantin Sagdeev ${ }^{1}$ (D), Sergey Melnikov ${ }^{1, *}$ (D), Mihail Isaev ${ }^{1}$ (D), Alexander Isaev ${ }^{1}$ (D) \\ 1. North-Caucasus Federal University - Institute of Digital Development - Infocommunication Department - Stavropol/Stavropol \\ Krai - Russian Federation. \\ *Correspondence author: territoreer@yandex.ru
}

\begin{abstract}
Objectives of this article are to create a method that should identify distortion of navigation signals using supplementary information obtained whilst processing the navigation parameters in the calculator of the navigation receiver. To develop this method, a comparative evaluation of the actual data of the navigation measurements with the calculated data values and a Bayesian probabilistic approach for detecting the navigation field distortion and identifying the interference. Test of results of the developed method showed that it enables a highly reliable assessment of the state of the navigation field and, if distorted, the identification of the distortion type: normal (undistorted) condition; abnormal state: when abnormally high errors appear spasmodically in the positioning estimation within individual measuring channels of the navigation receiver; slightly distorted state leading to a slow change in positioning accuracy; active spoofing manifested in the substitution of positioning coordinates. The article provides a new method for processing navigation parameters allowing to detect the fact of the navigation fields (NF) distortion and identify the interference effect on the navigation receiver (NR) of UAV.
\end{abstract}

Keywords: Positioning; Robotic automatically autopiloted vehicle; Distortion of the navigation field; Intentional interference; Processing of navigation parameters.

\section{INTRODUCTION}

The procedure for determining the navigation parameters for robotic unmanned aerial vehicles (UAV) using signals from global navigation satellite systems (GNSS) should ensure highest reliability (Venkatesh et al. 2017). However, in the environments where interference occurs, the measured estimates of the navigation parameters for the UAV will be significantly different from the true values. The instances where the errors exceed the maximum permissible values are particularly dangerous. These can be viewed as facts of distortion (violation of the integrity) of the navigation field (NF). The UAV control system should be promptly notified of the occurrence of such facts no later than within a fixed time, which is usually set as the moment of the next update of the navigation information; in other words, it should be ensured in real-time mode.

Received: Mar. 27, 2021 | Accepted: Sep. 27, 2021

Peer Review History: Single Blind Peer Review.

Section editor: Luiz Martins-Filho

This is an open access article distributed under the terms of the Creative Commons license. 
The analyzed sources (Binjammaz et al. 2016, Global navigation satellite system, Glonass 2008, 2016, Ivanov et al. 2015, Kim and Cho 2018, Layh and Gebre-Egziabher 2017, Pan et al. 2017, Rufa and Atkins 2016) describe various existing NF integrity monitoring systems designed to increase the reliability of the received navigation parameters. These are based on measuring redundancy and the use of additional measurement sources called functional supplements.

The algorithms and methods for monitoring the NF integrity which require a complex system consisting of space, ground and autonomous functional supplements are not acceptable for UAV in the autonomous flight mode. Due to the weight and dimensions restrictions of the UAV, only autonomous integrity control is acceptable, which renders to consumers the Aircraft Based Augmentations Systems (ABAS) using their own instruments installed on board.

The ABAS use measurement results from other on-board systems, such as a barometric altimeter, gyroscopic sensors, accelerometers and magnetic compass, as redundant data. For small UAV which use platformless inertial navigation systems (PINS), the existing ABAS is not very efficient as the gyroscopes and accelerometers used in PINS, based on microelectromechanical systems (MEMS), are characterized by increased noisiness. They also have high measurement error and cannot provide the required accuracy in time, more than few minutes.

The analysis also shows that the algorithms and methods for the autonomous monitoring of the NF integrity described in the sources (Binjammaz et al. 2016, Bishop 2006, Christophersen et al. 2006, Ioannides et al. 2016, Ivanov et al. 2015, Kim and Cho 2018, Layh and Gebre-Egziabher 2017, Onrubia et al. 2019, Pan et al. 2017, Rufa and Atkins 2016, Swamy 2017, Tran et al. 2018, Van den Bergh and Pollin 2019, Venkatesh et al. 2017, Wang et al. 2018, Yang et al. 2013) cannot be applied for the autonomous flight mode. They do not provide the required accuracy for determining the fact of the NF distortion and for identifying the type of interference.

Therefore, the aim of this article is to create a method allowing to intime and reliably detect distortion of NF and to identify the interference effects by using additional information obtained whilst processing the navigation parameters in the calculator of the navigation receiver (NR), yet without requiring any additional hardware due to the weight and size restrictions of the UAV.

The method is based on the combination of two approaches to processing navigation parameters:

- The approach described by Mazumder (2016), Wang et al. (2018), Onrubia et al. (2019), which involves a comparative assessment of the actual data of GNSS signals with the calculated data;

- Author's original approach, based on the application of the Bayesian method.

\section{METHOD DEVELOPMENT}

The navigation signals received to the input of the $\mathrm{NR}$, along with various types of noise and interferences, are known as random variables distributed according to a random law (Glonass 2016). Therefore, the error sequence for UAV positioning estimation obtained in the course of receiving radio navigation signals is also distributed randomly.

Where the signal-to-noise ratio $(h=S / J)$ at the input of the NR measuring channels exceeds the standard value set by the GNSS Interface Control Documents (Bishop 2006; Swamy 2017), which equals $45 \mathrm{dBm} \cdot \mathrm{Hz}^{-1}$, the only factor affecting the assessment accuracy of the navigation parameters is the additive noise at the NR input. In this case, the average values of the positioning estimation are closely equivalent to their true values, and minor errors fall within the specified confidence interval. This fact is the determining parameter of the NF being in a normal (undistorted) state.

Where additional intentional or unintentional disturbing influences occur, the average values of the positioning estimation prove to be different from the true values, and when their modules exceed some threshold values, such is interpreted as a fact of the violation of the NF integrity. The thresholds are to be set with account of ensuring the required confidence level values and allowing to identify the categories of interference.

In order to determine the fact of the NF distortion, the approach described in Mazumder (2016), Onrubia et al. (2019) and Wang et al. (2018) was applied. Essentially, it implies the polynomial approximation of an array of $N$ reliable measurements $R=(R 1$, $R 2 \ldots R j \ldots R N)$ in the form of estimates of the UAV positioning in the time interval $\mathrm{t} \epsilon\left[\mathrm{t}_{o}, \mathrm{t}_{a}\right]$ with a step $\tau$ equal to the period 
of receiving the navigation parameters. The approximation is performed by a polynomial of the $m$-th degree. Studies (Onrubia et al. 2019; Wang et al. 2018) have shown that using polynomials of the 5th or 6th degrees is sufficient to approximate the UAV trajectory with a set accuracy.

For the array of estimated coordinates of the UAV positioning, represented as a matrix-column R, the following system of equations has been obtained (Glonass 2016):

$$
V^{T} \times V \times C=V^{T} \times R
$$

where $\mathbf{V}$ is the Vandermonde matrix, size $N^{\prime}(m+1)$, where the elements are defined as follows: $V i, j=t_{i}^{j}(i=0 \ldots N-1) ;(j=0 \ldots m)$.

The $\mathbf{C}$ vector of the sought coefficients of the dimension polynomial $(m+1) \times 1$ is determined from the product of the equation:

$$
\mathrm{C}=\left(\mathrm{V}^{\mathrm{T}} \times \mathrm{V}\right)^{-1} \times \mathrm{V}^{\mathrm{T}} \times R
$$

As a result, the approximating function, which coincides with the distribution of reliable measurements of the positioning coordinates at $N+1$ points, looks as follows:

$$
F(t)=\sum_{j=0}^{m} C_{j} \cdot t^{j}
$$

An extrapolation procedure is applied to calculate the following positioning coordinate. By substituting the obtained coefficient values and the time value $t_{e}=t_{a}+\tau$ into the approximating polynomial, it is possible to obtain the value of the extrapolated function for the following coordinate reference at a given time interval, i.e.

$$
F\left(t_{e}\right)=\sum_{j=0}^{m} C_{j} \cdot t_{e}^{j}
$$

If $R$ measurements in $\mathrm{R}$ matrix column are uncorrelated and have the variance described by vector $\sigma_{R}^{2}$, the correlation matrix of errors of the polynomial coefficients is:

$$
\mathrm{K}_{\mathrm{C}}=\left(\mathrm{V}^{\mathrm{T}} \times \mathrm{V}\right)^{-1} \times \sigma_{\mathrm{R}}^{2}
$$

As a result, the task of determining the fact of the NF distortion is reduced to distinguishing between two hypotheses: a) whether the deviation of the estimated positioning coordinates from the calculated coordinate is the result of measurement errors or b) whether there exists an abnormal measurement which must be excluded from the processing.

It is most difficult to detect the fact of the NF distortion when the signal-to-noise ratio $h=\frac{S}{J}$ at the input of the NR measuring channels decreases slightly by $10 \ldots 20 \mathrm{dBm} \cdot \mathrm{Hz}^{-1}$, and two case scenarios are possible here. The first case is characterized by active imposition of false positioning coordinates to shift the UAV from the flight route or to force its landing. The second case is characterized by disturbance in the ionosphere, which causes fading due to the multipath reflections from the surrounding objects.

In both cases, the accuracy characteristics of the positioning are slightly distorted, which means they cannot be easily detected, since the average values of the positioning estimation are close to true ones. This imposes high standards of the sensitivity and reliability of the algorithms for detecting interference.

In order to eliminate such flaws, the proposed method involves a Bayesian inference to solving the problem with the NF distortion and identifying the interference effect (Christophersen et al. 2006; Ioannides et al. 2016). In this case, the variables characterizing the NF state are positioning estimates. The application of this approach is based on the calculation of the conditional probability of the occurrence of such an event as the current state $D k$ of the NF, provided the measurements obtained include a specific set of values of $R$ characteristics. The identification marks are the estimates of location $R=\left(R_{1}, R_{2} \ldots R_{j} \ldots R_{N}\right)$, while their 
average value $\mu_{R}$ and the $\sigma_{R}$ mean square errors (MSEs) are taken into account. Since these characteristics are random variables, the possibility of a specific representation of a random variable $R_{i}$ is characterized by the probability $P\left(R_{i}\right)$.

While the UAV is airborne, the NF can be in one of the $K$ states $D=\left(D_{1}, D_{2} \ldots D_{k} \ldots D_{K}\right)$. These states can be divided into the following typical classes:

- Normal (undistorted) state: orientation and navigation of the UAV receiver is performed in the normal mode by the integrated navigation system;

- Abnormal state, when abnormally high errors occur in the estimation of the positioning by the separate NR measuring channels. The UAV orientation and navigation are performed in the normal mode of the integrated navigation system; this module of the UAV spatial orientation parameters and inertial navigation systems (INS) parameters has a standard procedure for recurrent filtering of abnormal bursts;

- Slightly distorted state leading to a slow position change, whereby its assessment is possible with an acceptable error. The orientation and navigation are performed based on the navigation parameters of the integrated navigation system or of the INS, depending on the degree of distortion of the navigation field;

- Active spoofing, manifesting in either the substitution of the positioning coordinates for the smooth shift of the UAV from the set route or its forced landing;

- Energy suppression, when the signal-to-noise ratio over all NR channels drops sharply to a value lower than the sensitivity threshold: $13 \ldots 15 \mathrm{dBm} \cdot \mathrm{Hz}^{-1}$ (Kruschke and Liddell 2018), which leads to an abrupt and fast change of the positioning coordinates.

In order to give a probabilistic assessment of the NF state, when using a set of features of the obtained positioning sample, the Bayesian formula is used (Christophersen et al. 2006; Ioannides et al. 2016):

$$
p\left(\frac{D_{k}}{R_{j}}\right)=\frac{p\left(D_{k}\right) \cdot p\left(\frac{R_{j}}{D_{k}}\right)}{P(R)}=\frac{p\left(D_{k}\right) \cdot p\left(\frac{R_{j}}{D_{k}}\right)}{\int p(D) \cdot p\left(\frac{R_{j}}{D_{k}}\right) d D}
$$

where $p\left(D_{K} / D_{i}\right)$ is the a posteriori distribution density of the NF positioning in the $D_{k}$ state upon receipt of the set of features $R_{j}$, i.e., it is the value sought after to solve the problem of class recognition (here, distinguishing between the NF states); $p\left(R_{j} / D_{k}\right)$ is the a priori distribution density of the random variable $R_{j}$, provided the NF is in state $D k$. It has the meaning of the likelihood function and is determined by statistical processing of a sample of $N$ estimated positions, where the errors are distributed according to the normal law. In order to determine the likelihood function in a given confidence interval $( \pm 2 \sigma)$, a specific implementation of a set of factors $R_{j}$ is required to calculate their sample average $\mu_{R}$ and sample $\operatorname{MSE\sigma }_{R^{*}} p\left(D_{k}\right)$ is the a priori distribution density of a particular $D_{k}$ NF state determined by statistical processing of a sample of $N$ estimated positions, as the frequency of such events $N_{k}$, where the estimated positioning is included in the set confidence interval corresponding to the $N F$ state $D_{k}$, i.e., $P\left(D_{k}\right)=N_{k} / N$. $P(R)$ is the a priori probability of the appearance of a specific implementation of $R$ set of features for all possible NF states, used as the scaling factor equaling 1 for the general sample.

The statistically calculated likelihood function $p\left(R_{j} / D_{k}\right)$ allows to estimate the feasibility of the values of a specific implementation of the $R_{j}$ features contained in the sample of $N$ elements at a known a priori state of the navigation field $D_{k}$.

In order to ensure normal distribution, the sample range is set equal to $N \geq 30$. The formation time of such a sample upon receipt of the navigation parameters with a period of $0.1-0.5 \mathrm{~s}$ ranges from 3-15 s. This is a fairly long time, so this range is acceptable for a helicopter or multi-rotor UAV that can hover in one point. For an aircraft-type UAV, the sample range should be reduced to $N \leq 20$, applying Student's correction factors to determine the confidence interval.

Hence, after the statistical processing of the features, the application of the Bayesian approach allows to obtain an a posteriori distribution density of the possible probability values of NF positioning in one of the classes of its states. Indeed, by applying the Bayesian formula, an a priori distribution density established prior to the analysis of data from the received sample of features is transformed into an a posteriori one. This allows to evaluate the reliability of the NF state in a zone 
attributed to a particular class. Here, it should be taken into account that Eq. 6 is to be applied iteratively upon receiving each new data pack. Then the current distribution is considered a priori, and with the receipt of new data, a posteriori distribution is obtained, which becomes a priori for the next iteration. In addition, it should be observed that the application of the Bayesian approach requires additional time, which is spent on the NR calculator machine learning to statistically assess the current NF state based on the obtained characteristics in the form of the empirical distribution of the UAV positioning coordinates.

In order to obtain a straight confident a posteriori probability $P\left(D_{k} / D_{j}\right)$, the method of maximizing a posteriori density (MPD) is applied, which allows to find the point at which it is at maximum. Here, by logarithmizing the Bayesian formula, it is possible to obtain:

$$
\ln \left(p\left(D_{k} / R_{j}^{*}\right)\right)=\ln \left(p\left(D_{k}\right)\right)+\ln \left(p\left(R_{j} / D_{k}\right)\right)-\ln (P(R))
$$

Then the problem of maximizing Eq. 6 by the parameter $D_{k}$ will look as follows:

$$
L=\ln \left(p\left(R_{j} / D_{k}\right)\right)+\ln \left(p\left(D_{k}\right)\right) \rightarrow \max
$$

Equation 8 shows that this method maximizes the logarithms of the likelihood and the a priori probability distribution of a particular NF state. The second summate acts as a certain regulator of machine learning, since the greater the probability $P\left(D_{k}\right)$, the smaller the sample size, based on which a more accurate value of confidence probability can be obtained, and vice versa.

When applying the MPD method, the estimate of the current UAV positioning error arrives from the equation:

$$
\Delta R_{j}=R_{j}-F\left(t_{e}\right)
$$

Where $F\left(t_{e}\right)$ is the extrapolated value of the current positioning coordinates.

Assuming that the positioning errors are distributed according to the normal law, then

$$
\ln \left(p\left(R_{j} / D_{k}\right)\right)=\ln \left(\frac{1}{\sqrt{2 \pi \cdot \sigma_{k}^{2}}}\right) \cdot \exp \left(-\frac{\left(R_{j}-F\left(t_{e}\right)\right)^{2}}{2 \sigma_{k}^{2}}\right)=\ln =-\frac{1}{2} \ln \left(2 \pi \cdot \sigma_{k}^{2}\right)-\frac{\left(R_{j}-F\left(t_{e}\right)\right)^{2}}{2 \sigma_{k}^{2}}
$$

The first summate in Eq. 10 is a constant for the current distribution; therefore, the maximization of the likelihood logarithm is performed by minimizing the second summate. As a result, the task of maximizing the likelihood logarithm of the training sample is reduced to minimizing the quadratic error, i.e.

$$
\left(R_{j}-F\left(t_{e}\right)\right)^{2} \rightarrow \min
$$

Therefore, it was found that the larger the positioning error is, the more the likelihood function, shown in red in Fig. 1, moves the maximum of the a posteriori density, shown in blue, from the maximum of the a priori density (black dashed line). This means that the values of the errors of the UAV positioning coordinates can be used to assess the NF state, as they increase significantly under the influence of noise. As a result, a significant difference appears between the maxima of the a priori and a posteriori probability densities, which allows to introduce a threshold value by which one can establish the attribution of the NF state to one of the classes. 


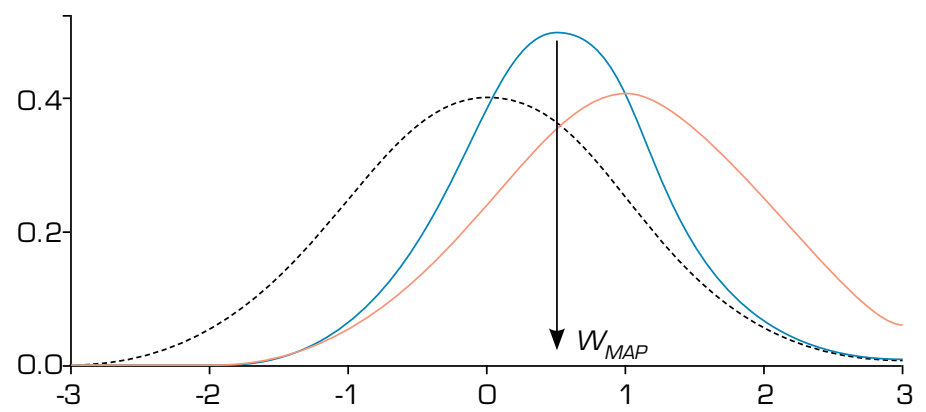

Figure 1. The transformation of the a priori density to the a posteriori density using the likelihood function.

Therefore, in order to decide on the fact of the NF distortion and the identification of the type of interference, threshold values are to be determined. Taking into account the normality of the distribution of the positioning estimation, based on the maximum likelihood method, the limiting MSE for the $\pm 2 \sigma$ confidence interval is established as the doubled minimum possible deviation for the current NF state.

The limiting MSE, which depends on the signal-to-noise ratio at the input of the NR measuring channels, is (Glonass 2016):

$$
\Delta_{k}=2 \cdot \operatorname{Pdop} \sqrt{(\delta t \cdot c)^{2}+\frac{(c)^{2}}{2 h_{k} \cdot F^{2}}}
$$

where $P d o p$ is the geometrical factor of accuracy dilution of positioning coordinates; $F$ effective width of the navigation signal spectrum (root mean square); $\delta t$ NR time error; $c$ speed of light.

In order to formulate a rule for deciding on the criterion of the MSE minimization, the following inequality is applied:

$$
\frac{1}{2} \ln \left(2 \pi \cdot \sigma_{k}^{2}\right)+\frac{\left(R_{j}-F\left(t_{3}\right)\right)^{2}}{2 \sigma_{k}^{2}} \leq \Delta_{k}
$$

Then, according to this criterion, to detect the fact of the NF distortion, inequality (Eq. 14) is to be checked, and to identify the types of interference, inequalities (Eq. 15) are to be analyzed, i.e.

$$
\begin{gathered}
\Delta R_{j} \leq \theta_{1}=\sqrt{2 \sigma_{k}^{2}\left(\Delta_{1}-0.5 \cdot \ln \left(2 \pi \sigma_{k}^{2}\right)\right)} \\
\Delta R_{j}>\theta_{k}=\sqrt{2 \sigma_{k}^{2}\left(\Delta_{k}-0.5 \cdot \ln \left(2 \pi \sigma_{k}^{2}\right)\right)}
\end{gathered}
$$

where $k=\overline{2, K}$.

Hence, if the positioning error exceeds the minimum threshold value $\theta_{1}$, the fact of NF distortion is obvious. Further comparison of the positioning error with other threshold values $\theta_{k}\left(l_{k}=2 \ldots_{K}\right)$ allows to identify the type of interference by attributing the NF state to a particular class.

Active spoofing is proposed to be detected during the UAV flight by hovering the UAV at one point in space (when detecting the NF state $D_{k} \in D_{s p}$ ). If the monitoring $h_{k} \geq h_{N} 45 \mathrm{dBm} \cdot \mathrm{Hz}^{-1}$ shows the normal NF state, but inequality (Eq. 14) is not satisfied, yet one of the inequalities (Eq. 15) is satisfied, such a situation should be identified as the active imposition of incorrect positioning coordinates. 
Mathematically, the condition of the fact of the active imposition of incorrect positioning looks as follows:

$$
D_{k} \in D_{s p} \text { if } h_{k} \geq h_{k} \cap \exists k=2 \ldots 5 ; \Delta R_{j}>\sqrt{2 \sigma_{k}^{2}\left(\Delta_{k}-0.5 \cdot \ln \left(2 \pi \sigma_{k}^{2}\right)\right)}
$$

Therefore, the complex of mathematical expressions described in this article, solved or satisfied in a certain sequence, demonstrate a method allowing to reliably detect the fact of the NF distortion and to identify its state using typical classes.

\section{RESEARCH RESULTS}

In order to test the developed method, a practical study was performed on the navigation receiver NEOM8N manufactured by U-BLOX. Based on the simulation of various types of interfering effects on the NR and the statistical processing of the navigation parameters, the following threshold values of the MSE have been established:

- $D_{1}=1.5 \times P \operatorname{dop}(\mathrm{m})$ normal (undistorted) NF state at $45 \mathrm{dBm} \cdot \mathrm{Hz}^{-1}$ (see Fig. 2a);

- $D_{2}=5 \times P \operatorname{dop}(\mathrm{m})$ : slightly distorted NF state at $=40 \ldots 33 \mathrm{dBm} \cdot \mathrm{Hz}^{-1}$, caused by the navigation signals reflection effect and, consequently, interference fading (see Fig. 2b);

- $D_{3}=8 \times P d o p(\mathrm{~m})$ : the distorted NF state at $=33 \ldots 28 \mathrm{dBm} \cdot \mathrm{Hz}^{-1}$ caused by ionospheric disturbances (see Fig. $2 \mathrm{c}$ );

- $D_{4}=30 \times P d o p(\mathrm{~m})$ : increasing deliberate interference at $=28 \ldots 15 \mathrm{dBm} \cdot \mathrm{Hz}^{-1}$ (see Fig. $2 \mathrm{~d}$ );

- $D_{5}=50 \times P d o p(\mathrm{~m})$ : spasmodic energy suppression at $=20 \ldots 10 \mathrm{dBm} \cdot \mathrm{Hz}^{-1}$ (see Fig. $2 \mathrm{e}$ and $2 \mathrm{f}$ ).

Figure 2a demonstrates an example of the distribution of the UAV positioning errors for the normal NF state, without distortions. Parameters of this state are $P d o p=1$, signal-to-noise ratios minimum $45 \mathrm{dBm} \cdot \mathrm{Hz}^{-1}$, and time errors $\sigma t=1 \ldots 2 \mathrm{~ns}$. Under these conditions, the horizontal coordinate errors $<0.9 \mathrm{~m}$ and the calculated limiting MSE $\Delta_{1}$ is $1.5 \mathrm{~m}$. Therefore, if the error of the UAV positioning has a distribution falling into the required confidence interval $\pm 2 \sigma$ with a probability of 0.95 , it should be assumed, with a confidence probability of 0.95 , that the NF is in the normal state.

Initial training should be performed at the initial location of the UAV after the NR transition to the tracking mode, provided that the NF is in the normal state $D_{n}$.

Examples confirming the possibility of implementing the procedure for identifying interference effects are shown in Fig. $2 b-e$. As soon as the positioning error exceeds one of the threshold values, according to the established criterion $\theta_{k}$, a decision is made on the type of interference effect.

The retraining procedure is performed after determining the fact of NF distortion during its transition from one state to another. In this case, the confidence probability of finding the NF in the previous state is significantly reduced, which leads to lower reliability. In the case of a distorted (suppressed) NF state, the likelihood function that has changed and shifted along the abscissa axis even more significantly shifts the maximum of the a posteriori density from the maximum of the a priori density (Fig. 1).

The data presented in Fig. 2 was obtained by manual processing u-blox 9 receiver data. There were analyzed about 600 flights, which contain shown type of distortions/suppressions.

Figure $2 \mathrm{~b}$ shows the NF distortion occurs due to interference fading. Signal parameters is $P d o p=1 \ldots$... signal-to-noise ratios: $40 \ldots 33 \mathrm{dBm} \cdot \mathrm{Hz}^{-1}$, time errors $=2 \ldots 4 \mathrm{~ns}$. It is apparent that the NF passes from a normal state limited by a radius of $1.5 \mathrm{~m}$ to a distorted state, then returns to the normal state for a while, and then goes into a distorted state again. Therefore, retraining is also performed after the field returns to its normal state (after the distorting effects or interference effects discontinue). The retraining procedure should be performed in the UAV hovering mode for as long as the training sample is formed, which allows to determine, with a probability of 0.95 , that the NP is in the new state.

Figure $2 c$ demonstrates an example of the distribution error of the UAV positioning when the NR is in a distorted field caused by ionosphere. $P d o p=1 \ldots 2$, signal-to-noise: $33 \ldots 28 \mathrm{dBm} \cdot \mathrm{Hz}^{-1}$, time errors $=2 \ldots 5 \mathrm{~ns}$.

On Fig. 2d, UAV approaches the source of intentional interference. $P d o p=1 \ldots$, signal-to-noise: $45 \ldots 25 \mathrm{dBm} \cdot \mathrm{Hz}{ }^{-1}$, time errors $=2 \ldots 6 \mathrm{~ns}$. 
On Fig. 2e, UAV falls into the zone of energy suppression. $P d o p=1 \ldots 3.5$, signal-to-noise: $45 \ldots 10 \mathrm{dBm} \cdot \mathrm{Hz}^{-1}$, time errors $=$ $2 \ldots 23 \mathrm{~ns}$.

On Fig. 2f, UAV falls into complete energy suppression with a subsequent restoration of the NF. $P d o p=1 \ldots 3.5$, signal-to-noise ratio in the measuring channels, first decreasing $45 \ldots-10 \mathrm{dBm} \cdot \mathrm{Hz}^{-1}$, and then increasing $-10 \ldots 40 \mathrm{dBm} \cdot \mathrm{Hz}^{-1}$, and time errors from 2 to $500 \mathrm{~ns}$ and from 500 to $5 \mathrm{~ns}$.
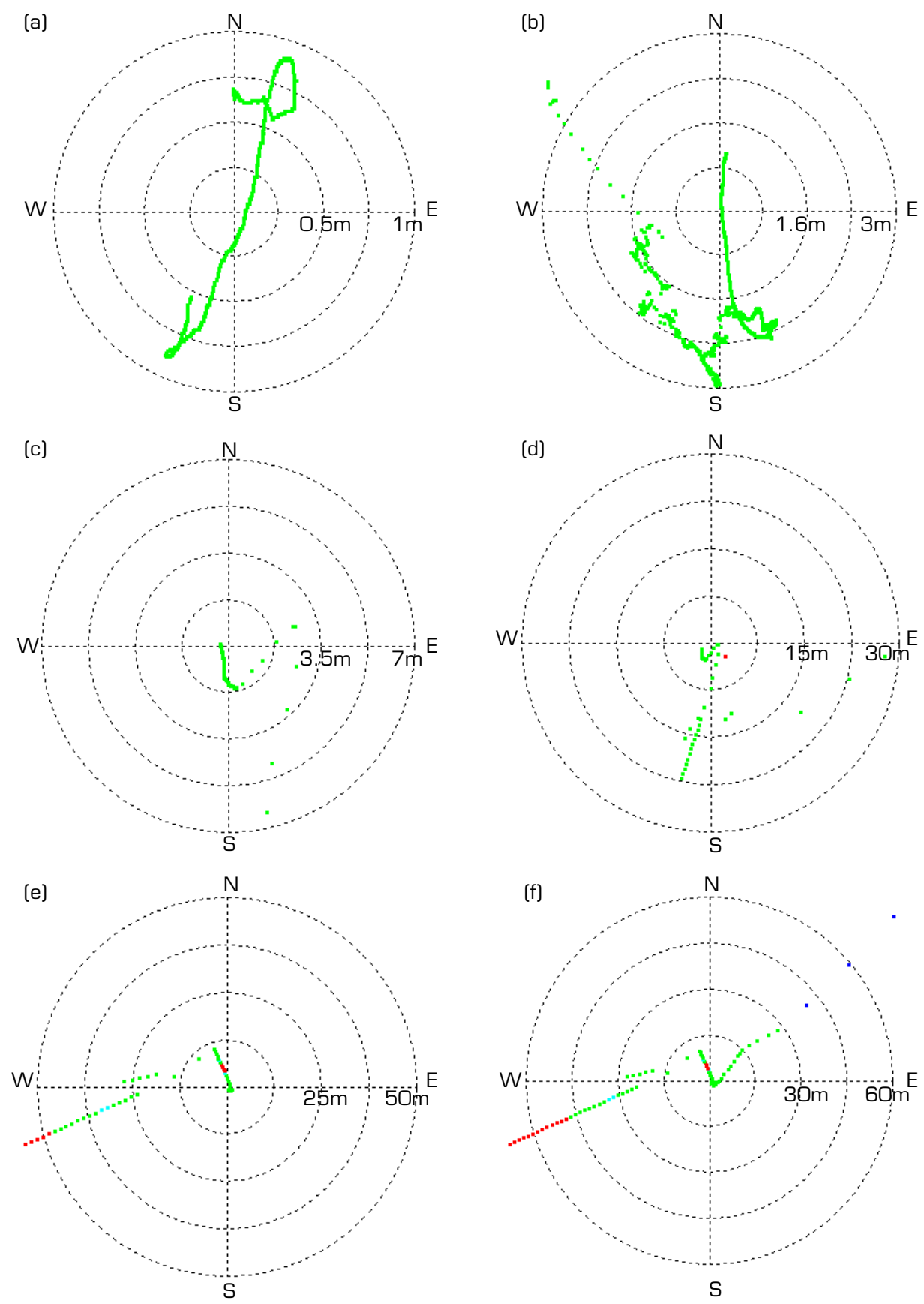

Figure 2. Examples of the distribution of the UAV positioning error in different NF states. The dotted line signifies the delay in receiving Global Positioning System/Global Navigation Satellite System (GPS/GLONASS) packages. Red dots mean absence of a reception signal. Blue dots show weak signal reception. 
The implemented requirements for the simulation model are listed below. The simulation model should estimate the current NF state according to the obtained UAV positioning coordinates; make highly reliable assessment of the occurrence or absence of NF distortions; in case of existing NF distortions, detect the type of interference impact by attributing it to one of the typical classes: normal (undistorted) state; abnormal state, when abnormally big errors in positioning estimates randomly arise in separate measurement channels of the navigational receiver; insignificantly distorted state which leads to slow positioning shift; active spoofing, evident in positioning coordinates substitution; energetic suppression of GPS/GLONASS signals.

The input parameters of simulation model include: Pdop (geometric factor of positioning coordinate accuracy degradation); navigation $N$-UAV positioning coordinates set; navigation receiver time error; signal-to-noise ratio.

The limitations include: sample size $\mathrm{N} \geq 30$ (Mazumder 2016, Kruschke and Liddell 2018); number of NF states (typical classes), equal to 5 .

The output parameters include: confidence signal for the decision taken $S_{c}$; GNSS distortion signal $S_{d}$; identifying code for interference impact class $S_{k}$; active spoofing detection signal $S_{s p}$.

For the block diagram of a simulation model, see Fig. 3.

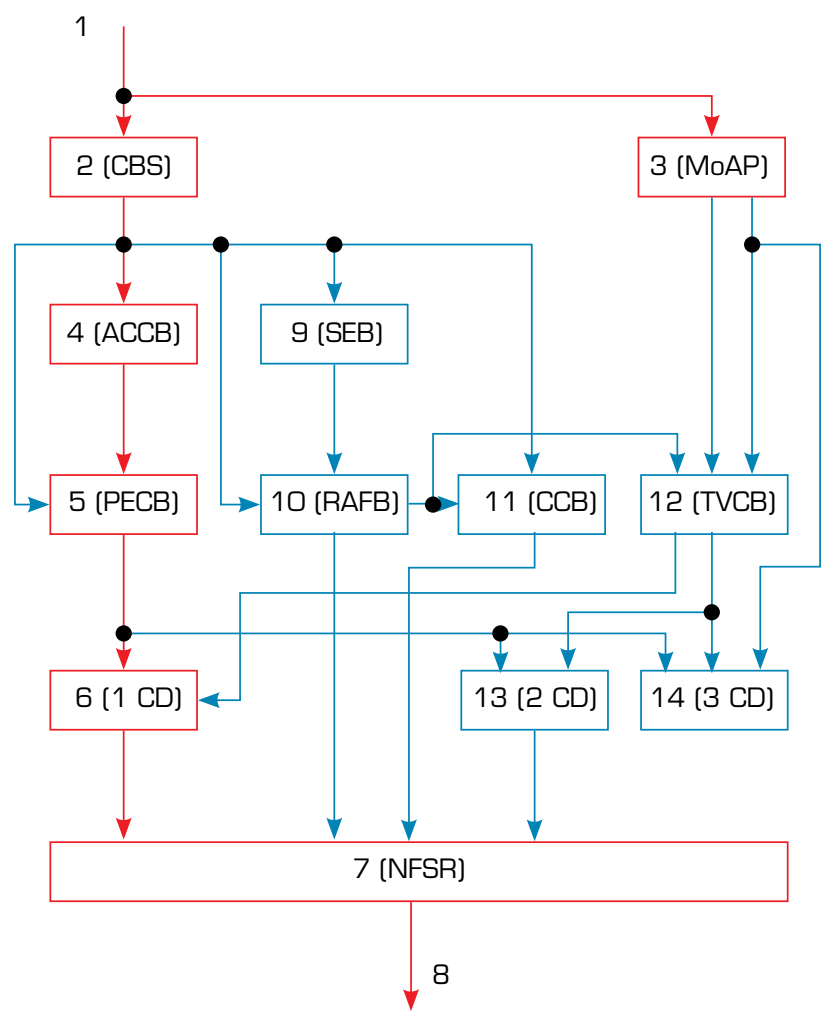

Figure 3. Block diagram of a simulation model for determining navigational field distortions and identifying interference impact.

$\mathrm{CBS}=$ coordinates buffer store; $\mathrm{ACCB}=$ approximation coefficients computation block; $\mathrm{SEB}=$ statistical estimation block; $\mathrm{PECB}=$ positioning error computation block; $\mathrm{RAFB}$ = recursive anomaly filtering block; $\mathrm{CCB}=$ coefficient computation block; $\mathrm{TVCB}=$ threshold values computation block; $1 \mathrm{CD}=$ first computing device; $2 \mathrm{CD}=$ second computing device; $3 \mathrm{CD}=$ third computing device

Coordinates buffer store (CBS-block 2) is a static memory device for recording and storing a sample of $\mathrm{N}$ values of positioning coordinates $R_{y}$, each with $p$ digits. The sample is sent through input bus 1 . Binary codes of positioning coordinates are sent across the inner bus from this memory to the approximation coefficients computation block (ACCB-block 4), current UAV positioning error computation block (PECB-block 5), statistical estimation block (SEB-block 9), recursive anomaly filtering block (RAFBblock 10), and confidence coefficient computation block (CCB-block 11). 
A buffer memory of additional parameters (MoAP-block 3) is a static type memory device used for recording and storing current parameters characterizing the NF state: geometric factor of positioning coordinate accuracy degradation $P d o p$; navigation receiver time error $\delta t$; signal-to-noise ratio $h_{k}$ at the navigation receiver measurement channels input. It should also contain a demultiplexer for delivering the stored data to one of block 3 (MoAP) outputs. Moreover, Pdop and $\delta t$ values are sent to the first input of MoAP (block 3 ), and $h_{k}$ values are sent to the second input.

A computation block for approximation coefficients (ACCB-block 4) is based on a field-programmable gate array (FPGA). Its configuration was logically synthesized and loaded into the FPGA, which ensures performance of the following functional tasks: receiving and storing a vector of $\mathrm{N}$ positioning coordinate values $R_{y}$; forming $t \epsilon\left[t_{0}, t_{a}\right]$ for the current times at $\tau$, pace equal to the arrival period of navigation parameters, Vandermonde matrix size $N \times(m+1)$, with elements set as $\mathbf{V}_{\boldsymbol{i}, \boldsymbol{j}}=t_{i}^{j}(i=0 \ldots N-1 ; \mathrm{j}=0 \ldots \mathrm{m})$, where $m$ is the degree of the 5-degree polynomial approximant (Mazumder 2016, Kruschke and Liddell 2018); computation of C vector of polynomial approximant of $(m+1) \times 1$ dimension coefficients by solving the equation:

$$
\mathrm{C}=\left(\mathrm{V}^{\mathrm{T}} \times \mathrm{V}\right)^{-1} \times \mathrm{V}^{\mathrm{T}} \times \mathrm{R}
$$

An error computation block (PECB-block 5) is based on the FPGA by logically synthesizing and loading its configuration in the pattern, which ensures the performance of the following functional tasks: receiving and storing current positioning coordinates $R_{y}$; receiving and storing vector $\mathrm{C}$ of $m+1$ polynomial approximant coefficient values; computation of the next positioning coordinate for the moment $t_{e}=t_{a}+\tau$ through the extrapolation procedure:

$$
F\left(t_{e}\right)=\sum_{j=0}^{m} C_{j} \cdot t_{e}^{j}
$$

Error computation for the current UAV position $\Delta R_{y}=R_{y}-F\left(t_{e}\right)$.

The purpose of the first calculating device (1 CD-block 6) is making decisions about existence or absence of NF distortion by comparing the calculated errors of current UAV positioning with the minimal threshold value $\theta_{1}$. If the positioning error exceeds the threshold value $\theta_{1}$, i.e., $\Delta R_{y}>\theta_{1}$, it signifies NF distortion. The device outputs GNSS distortion signal $S_{u}$, which equals 1 . Otherwise, the NF is marked as being in the normal (undistorted) state, and $S_{u}$ signal equals 0.

The purpose of the navigation field state register (NFSR-block 7) is recording and sending the following signals through output bus 8: confidence signal $S_{e}$, GNSS anomaly signal $S_{a}$, GNSS distortion signal $S_{d}$, identifying code for interference impact class $S_{k}$, and active spoofing signal $S_{s p}$. These signals are recorded in the appropriate register cells.

A statistical assessment block (SEB-block 9) is based on a field-programmable gate array (FPGA). Its configuration is logically synthesized and loaded to FPGA, which ensures the performance of the following functional tasks: receiving and storing a sample of $\mathrm{N}$ positioning coordinate values $R_{y}$; calculating their sample mean $\mu_{R}$ and sampling variance $\sigma_{R}^{2}$.

A RAFB (block 10) is designed for filtering and screening abnormal surges, i.e., significant brief positioning errors occurring at random times. It is built based on FPGA by logically synthesizing and loading its configuration into the FPGA, which ensures the performance of the following functional tasks: receiving and storing a sample of $\mathrm{N}$ positioning coordinate values $R_{y}$, and current values of sampling mean $\mu_{R}$ and sampling variance $\sigma_{R}^{2}$; recursive filtering of sampling mean $\mu_{R}$ and sampling variance $\sigma_{R}^{2}$ of positioning coordinates by performing such procedures, as

$$
\begin{gathered}
\mu_{R}(t)=\mu_{R}(t-1)+\frac{1}{N}\left(R_{y}(t)-\mu_{R}(t-1)\right)=\mu_{R}(t-1)+\frac{1}{N} \Delta R_{y}(t) \\
\sigma_{R}^{2}(t)=\sigma_{R}^{2}(t-1)+\frac{1}{N}\left(\left(\Delta R_{y}(t)\right)^{2}-\sigma_{r}^{2}(t-1)\right)
\end{gathered}
$$


checking the validity of: $\left(\Delta R_{y}(t)\right)^{2}>\sigma_{R}^{2}(t-1)$, and if the function is true, the current coordinate is considered distorted and substituted by a sampling mean value. At the same time, a GNSS anomaly signal $S_{a}$ equal to 1 is generated, otherwise the signal equals zero.

The purpose of a confidence CCB (block 11) is to compute the confidence coefficient $P\left(D_{k} / D_{y}\right)$ for NF in the current state $D_{k}$. It is performed by logically synthesizing and loading its configuration to FPGA. This configuration ensures the performance of the following functional tasks: receiving and storing a sample of $\mathrm{N}$ positioning coordinate values $\mathrm{R}_{y}$, and sampling mean $\mu_{R}$ and sampling variance $\sigma_{R}^{2}$; computing likelihood function (Mazumder 2016) $p\left(R_{y} / D_{k}\right)$ for confidential interval $\pm 2-\sigma$ according to Eq. 21 :

$$
p\left(\frac{R_{y}}{D_{k}}\right)=\frac{1}{\sqrt{2 \pi \cdot \sigma_{R}^{2}}} \cdot \exp \left(-\frac{\left(R_{y}-F\left(t_{e}\right)\right)^{2}}{2 \sigma_{R}^{2}}\right)
$$

calculating a priory probability $P\left(D_{k}\right)$ as a frequency of an event $N_{k}$, at which the estimated positioning is within the set confidence interval $\left(\mu_{R-2 \sigma_{R \leq R y \leq u R+2 s R}}\right)$, corresponding to NA state $D_{k}$, i.e., $P\left(D_{k}\right)=N_{k} / N$; determining confidence coefficient $P\left(D_{k} / R_{y}\right)$ for NF in the current state $D_{k}$ according to the equation $P\left(D_{k} / R_{y}\right)=P\left(D_{k}\right) \bullet p\left(R_{y} / D_{k}\right)$; generating confidence signal $S_{e}$, which equals 1 , where the confidence coefficient exceeds or equals the set value ( 0.95 for confidential interval $\pm 2-\sigma)$, otherwise, the signal equals zero.

The purpose of the threshold values computation block (TVCB-block 12) is calculating threshold values, which classify a typical NF state. Its configuration is logically synthesized and loaded into FPGA. This configuration ensures the performance of such functional tasks as: receiving the current sampling dispersion $\sigma_{R}^{2}$ of positioning coordinates from block 10 (RAFB) and storing them; receiving current values $P d o p, \delta_{\mathrm{t}}$ and $h_{k}$ from memory block 3 (MoAP) and storing them; calculating the ultimate MSE which depends on signal and noise environment in the NF measurement channels:

$$
\Delta_{k}=2 \cdot \operatorname{Pdop} \sqrt{(\delta t \cdot c)^{2}+\frac{(c)^{2}}{2 h_{k} \cdot F^{2}}}
$$

calculating threshold values for each $k$ class of interference impact:

$$
\theta_{k}=\sqrt{2 \sigma_{R}^{2}\left(\Delta_{k}-0.5 \ln \left(2 \pi \sigma_{R}^{2}\right)\right)}
$$

The second computing device (2CD-block 13) is designed for identifying typical interference impacts on NP except for active spoofing. It generates a code which identifies the interference impact class $S_{k}$ according to comparison of the calculated UAV positioning error with the threshold value for each interference class, i.e., $\Delta R_{y}>\theta_{k}$. The 2CD contains $k$ comparison circuits according to the number of identified typical classes of interference impact, and the encryption unit for presenting the interference type in binary code.

The third computing device (3CD-block 14) is designed for identifying active spoofing by checking the following conditions: $D_{k} \in D_{s p}$ if $h_{k} \geq h_{H} \cap \Delta R_{y>\theta_{k}}$.

Fulfilling these conditions means that interference impact is of the active spoofing class, if the current UAV positioning error in the normal NF state exceeds the acceptable threshold for the undistorted NF state. Here, the computing device generates active spoofing present signal $S_{s p}$.

The 3CD (block 14) contains $k+\mathrm{K}$ comparison circuits, where $k$ is the number of identifiable typical classes of interference impact, $K$ is the number of NF measurement channels included in calculating the current positioning coordinate (as a rule, six channels are used), and logical elements, producing a solution. 
The described device is designed to work as part of the UAV navigation receiver controlled by its calculator. The device acts as a coprocessor implementing a technology for processing navigational parameters to detect NF distortions and identify the interference impact.

Modern technology allows configuring the approximation coefficients computation block (ACCB-block 4), positioning error computation block (PECB-block 5), SEB (block 9), RAFB (block 10), confidence CCB (block 11), and TVCB (block 12) in one FPGA. Having the above-listed blocks in one FPGA significantly simplifies their interaction process (Wang et al. 2018), and, therefore, the control process performed by the calculator of the navigation receiver. Also, such solution ensures compliance with the weight and size limitation requirements of the navigation receiver.

Based on the simulation model block diagram (Fig. 3), the simulation model was developed in MathLab (v. 14) environment. Blocks 2 (CBS) and 3 (MoAP) of the block diagram are included in block 1; block 10 (RAFB) is merged with block 11 (CCB). Other simulation model blocks are in line with the block diagram. The developed simulation model is shown in Fig. $4 \mathrm{a}$. Figure $4 \mathrm{~b}$ shows the example of input data obtained from an UAV navigational receiver, where index is a number of the input package, SV C/N0 is a signal-to-noise ratio, GPS time is navigation receiver time, coordinated universal time (UTC) is current time $\left(\delta_{\mathrm{t}}\right.$ computed based on GPS time and UTC parameters).

(a)

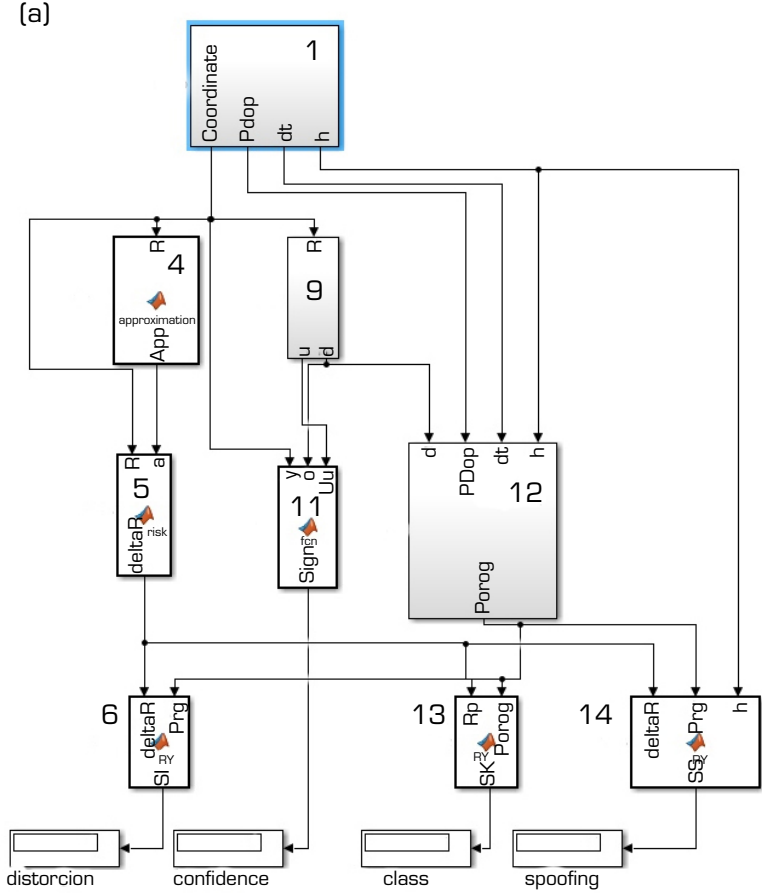

(b)

\begin{tabular}{|c|c|c|c|c|}
\hline Index & PDOP & SVC/NO & GPS time & UTC \\
\hline 1427 & 100.0 & & $2032: 389 \ldots$ & $12: 08: 11 \ldots$ \\
\hline 1428 & 100.0 & & $2032: 389 \ldots$ & $12: 08: 11 \ldots$ \\
\hline 1429 & 100.0 & & $2032: 389 \ldots$ & $12: 08: 11 \ldots$ \\
\hline 1430 & 100.0 & & $2032: 389 \ldots$ & $12: 08: 11 \ldots$ \\
\hline 1431 & 100.0 & & $2032: 389 \ldots$ & $12: 08: 11 \ldots$ \\
\hline 1432 & 100.0 & & $2032: 389 \ldots$ & $12: 08: 12 \ldots$ \\
\hline 1433 & 100.0 & & $2032: 389 \ldots$ & $12: 08: 12 \ldots$ \\
\hline 1434 & 100.0 & & $2032: 389 \ldots$ & $12: 08: 12 \ldots$ \\
\hline 1435 & 100.0 & & $2032: 389 \ldots$ & $12: 08: 12 \ldots$ \\
\hline 1436 & 100.0 & & $2032: 389 \ldots$ & $12: 08: 12 \ldots$ \\
\hline 1437 & 100.0 & & $2032: 389 \ldots$ & $12: 08: 13 \ldots$ \\
\hline 1438 & 100.0 & & $2032: 389 \ldots$ & $12: 08: 13 \ldots$ \\
\hline 1439 & 100.0 & & $2032: 389 \ldots$ & $12: 08: 13 \ldots$ \\
\hline 1440 & 100.0 & & $2032: 389 \ldots$ & $12: 08: 13 \ldots$ \\
\hline 1441 & 100.0 & & $2032: 389 \ldots$ & $12: 08: 13 \ldots$ \\
\hline 1442 & 100.0 & & $2032: 389 \ldots$ & $12: 08: 14 \ldots$ \\
\hline 1443 & 100.0 & & $2032: 389 \ldots$ & $12: 08: 14 \ldots$ \\
\hline 1444 & 100.0 & & $2032: 389 \ldots$ & $12: 08: 14 \ldots$ \\
\hline 1445 & 100.0 & & $2032: 389 \ldots$ & $12: 08: 14 \ldots$ \\
\hline 1446 & 100.0 & & $2032: 389 \ldots$ & $12: 08: 14 \ldots$ \\
\hline 1447 & 100.0 & & $2032: 389 \ldots$ & $12: 08: 15 \ldots$ \\
\hline 1448 & 100.0 & & $2032: 389 \ldots$ & $12: 08: 15 \ldots$ \\
\hline 1449 & 100.0 & & $2032: 389 \ldots$ & $12: 08: 15 \ldots$ \\
\hline 1450 & 100.0 & & $2032: 389 \ldots$ & $12: 08: 15 \ldots$ \\
\hline 1451 & 100.0 & & $2032: 389 \ldots$ & $12: 08: 15 \ldots$ \\
\hline 1452 & 100.0 & 21.00 & $2032: 389 \ldots$ & $12: 08: 16 \ldots$ \\
\hline 1453 & 100.0 & 21.00 & $2032: 389 \ldots$ & $12: 08: 16 \ldots$ \\
\hline 1454 & 100.0 & 19.67 & $2032: 389 \ldots$ & $12: 08: 16 \ldots$ \\
\hline
\end{tabular}

Figure 4. Developed simulation model. (a) Structure of model in MatLab environment; (b) Example of simulation model input data.

The output parameters of the simulation model in Fig. 2a include: confidence signal $S_{c}$ (field "confidence"), GNSS distortion signal $S_{D}$ (field “distortion"), interference impact class $S_{C l}$ (field "class"), active spoofing presence signal $S_{s p}$ (field "spoofing”).

The results of comparing the above method and manual data processing are shown in Figs. 5, 6 and 7. Manual data processing was performed using the u-center program (v19.04).

Figure 5 shows the process of suppressing the GPS/GLONASS signal and changing the deviation of the UAV positioning. The $P d o p$ value is indicated by red dots in Fig. 5. The data of the suppressed GPS/GLONASS navigation receiver was loaded into the simulation model. Figures 5 and 6 show the results of simulation model and determine the fact of energy suppression of the GPS/GLONASS signal. Without the distortion, Fig. 5, information field "distortion" has value zero. Further, when the receiver was subjected to short-term suppression, the simulation model detected and established 
suppression of navigation field, value "distortion" changed to 1, the "type" field to 4, which corresponds to intentional signal suppression (Figs. 6 and 7). Therefore, the developed method adequately describes the fact of the navigation field distortion and identifies its state.

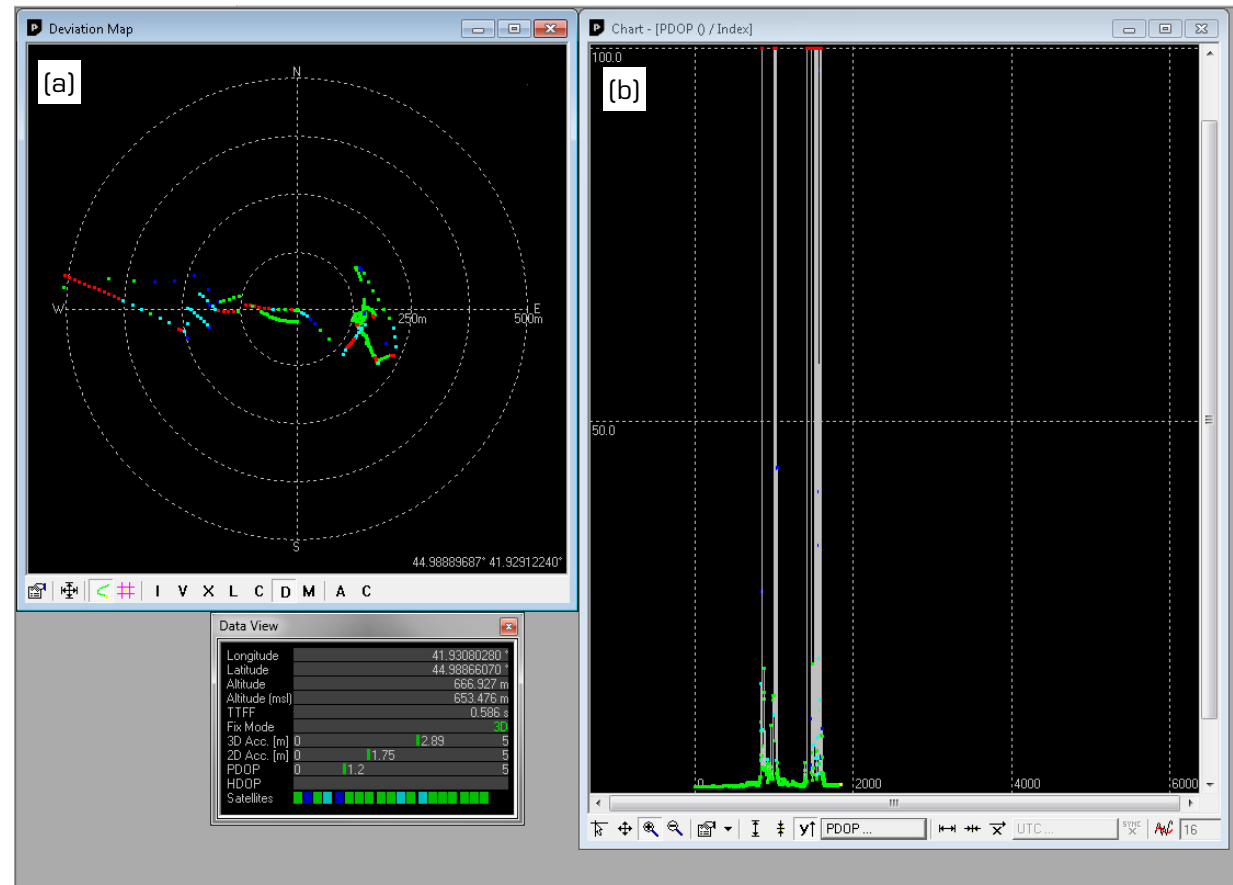

Figure 5. Deviation in UAV positioning and Pdop parameters when suppressing the GPS/GLONASS signal.

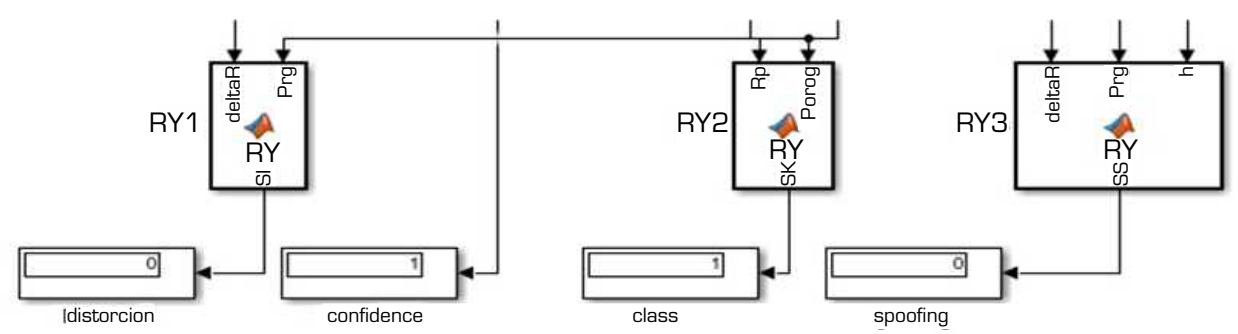

Figure 6. Result of processing GPS/GLONASS packets in an undistorted navigation field.

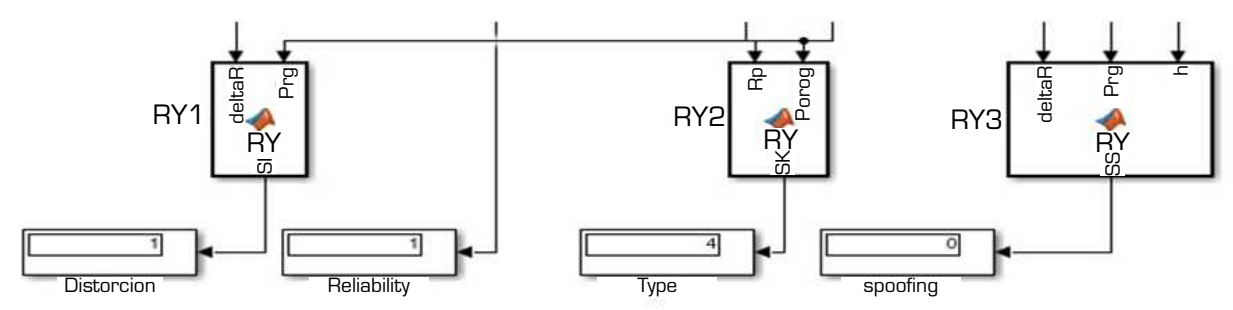

Figure 7. Result of GPS/GLONASS signal suppression.

The full model created by MatLab is presented in Fig. 8.

The algorithm based on the developed method was integrated into the UAV controlling algorithm. In order to evaluate the quality of the algorithm performance, a software-hardware system of simulation modeling was used (Fig. 8). 


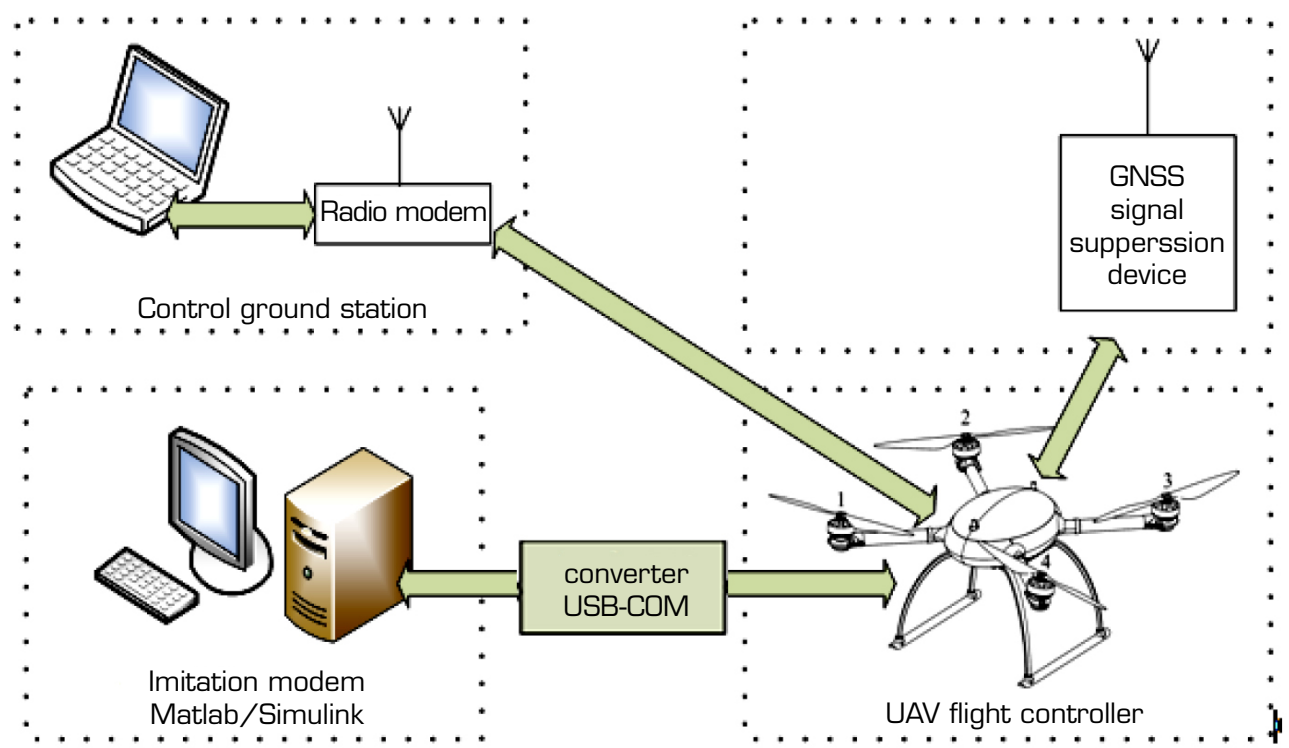

Figure 8. Software-hardware system of simulation modeling.

MatLab Simulink environment has created the following program models: brushless motor, propeller, atmosphere, power battery. The brushless motor model based on the blade elements provides data on the thrust created and the moment of resistance to rotation, depending on the air velocity. The brushless electric motor and propeller models are matched to the bench characteristics of real items. The atmosphere model simulates various environmental conditions, providing the ability to vary the atmospheric parameters, from flight altitude to wind exposure. The power battery parameters (voltage, capacity, internal resistance) can be changed by the user.

For testing, a 6-kg quadrocopter UAV was used. Atmospheric pressure $703 \mathrm{mmHg}$, air temperature $25^{\circ} \mathrm{Celsius,} \mathrm{humidity} 60 \%$, wind speed $1 \mathrm{~m} \cdot \mathrm{s}^{-1}$. Global navigation satellite systems suppression started after $2 \mathrm{~min}$ in cruising flight.

The program model data are entered in the flight controller through the interface converter from Universal Serial Bus to Recommended Standard 232 port (USB-RS232). The UAV flight controller changes the onboard sensors data to the data obtained from the imitation model. The GNSS signal suppression device creates interference, which distorts the navigation field or completely suppresses the signal. This device allows to imitate the shift of the UAV positioning coordinates through smooth change of the interference impact level.

Hence, the hardware and software complex allows to test the algorithms for controlling the spatial orientation and trajectory flight control of the UAV, the logic of the flight controller in various situations, namely: the absence and distortion of the NF, up to the complete failure of the satellite navigation system reception channel, taking into account the discharge of the power battery, in manual or automatic control mode, etc. The tests were performed under various conditions that may occur during the flight.

The simulation results in the form of the time dependence of the positioning errors of the UAV in the autonomous mode are shown in Fig. 9. The error in the position estimate was calculated as the difference between the position estimate performed by the PINS using inertial meters and the ideal position estimate obtained from the simulation model of the UAV.

Flight test show that, after distortion GPS/GLONASS signals, UAV will have better positioning accuracy (Fig. 9). Positioning error increased by increasing MEMS errors.

Based on the test results, it can be concluded that the algorithm of the method for identifying the fact and type of distortion of the navigation field functions correctly, switching to the appropriate flight modes. 


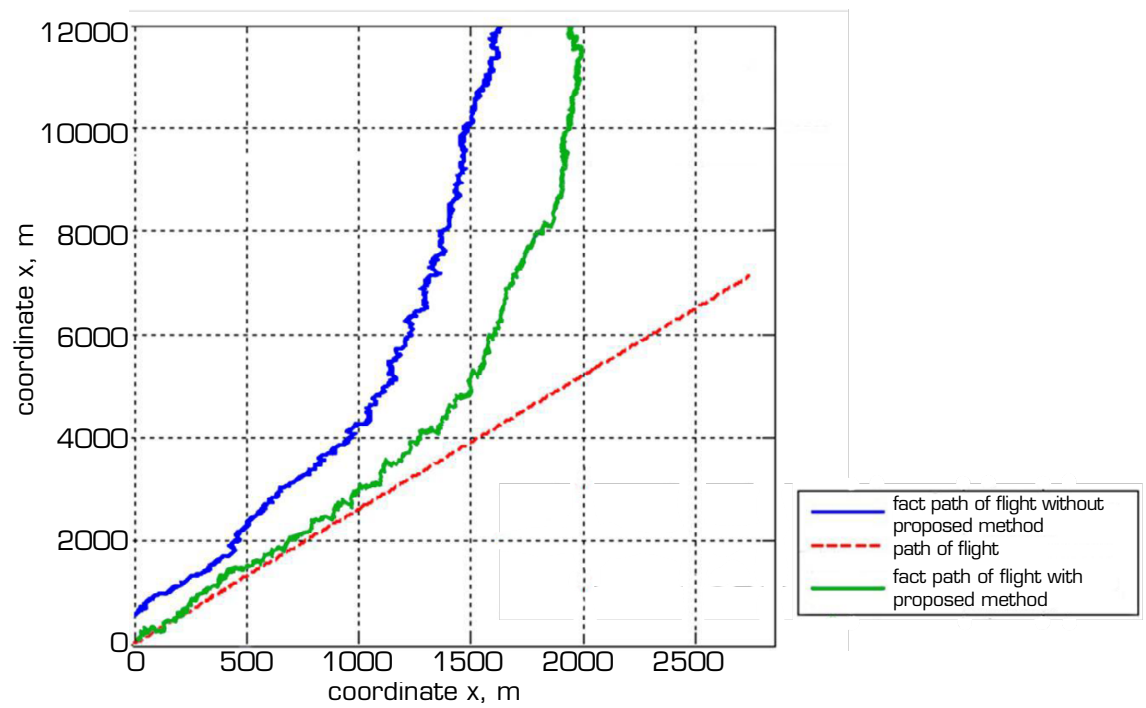

Figure 9. Positioning errors of the UAV in the autonomous mode.

\section{CONCLUSION}

The article provides a new method for processing navigation parameters allowing to detect the fact of the NF distortion and identify the interference effect on the NR installed onboard an UAV.

The practical implementation of the developed method in the NR allows to: evaluate the current state of the NF by the received coordinates of the UAV positioning with a confidence probability of 0.95 ; make a reliable decision on the actual presence or absence of the NF distortion according to the MPD criterion; identify the type of interference by assigning it to one of the typical classes, where the fact of the NF distortion is established; switch to navigation data received from the autonomous UAV control system of the PINS upon detecting the fact of active spoofing and energy suppression of GPS/GLONASS; and set the threshold values for the errors of the UAV positioning for the current signal-noise situation.

When changing the signal-noise environment, this method enables a short-term retraining of the NR.

The advantage of the developed method, unlike the previous ones, is the possibility of using it to improve the UAV control algorithm by expanding its functionality in the autonomous operation mode in the environments with natural and deliberate interference.

\section{AUTHORS' CONTRIBUTION}

Conceptualization: Sagdeev K and Linets G; Formal Analysis: Sagdeev K; Funding Acquisition: Isaev M; Investigation: Sagdeev K and Melnikov S; Methodology: Sagdeev K; Project Administration: Linets G; Software: Isaev A and Isaev M; Supervision: Linets G; Validation: Isaev A, Isaev M, Melnikov S; Writing - Original Draft: Sagdeev K, Linets G, Melnikov S; Writing - Review and Editing: Sagdeev K, Linets G, Melnikov S.

\section{DATA AVAILABILITY STATEMENT}

Data sharing is not applicable due to funding. 


\section{FUNDING}

Russian Foundation of Basic Research

Grant No. 20-37-90010

\section{REFERENCES}

[Glonass] Global navigation satellite system (2008) Interface control document: Navigational radiosignal in bands L1, L2. 5.1 ed. Moscow: Glonass. [accessed Nov. 9 2021]. https://russianspacesystems.ru/wp-content/uploads/2016/08/ICD_ GLONASS_eng_v5.1.pdf

[Glonass] Global Navigation Satellite System (2016) Interface Control Document: Code Division Multiple Access Open Service Navigation Signal in L1 frequency band. 1.0 ed. Moscow: Glonass. [accessed Nov. 92021]. https://russianspacesystems. ru/wp-content/uploads/2016/08/ICD-GLONASS-CDMA-L1.-Edition-1.0-2016.pdf

Binjammaz TA, Al-Bayatti AH, Al-Hargan AH (2016) Context-aware GPS integrity monitoring for intelligent transport systems. J Traffic Transp Eng 3(1):1-15. https://doi.org/10.1016/j.jtte.2015.09.002

Bishop CM (2006) Pattern recognition and machine learning. New York: Springer.

Christophersen HB, Pickell RW, Neidhoefer JC, Koller AA, Kannan SK, Johnson EN (2006) A compact guidance, navigation, and control system for unmanned aerial vehicles. J Aerosp Comput InfCommun 3(5):187-213. https://doi.org/10.2514/1.18998

Ioannides RT, Pany T, Gibbons G (2016) Known vulnerabilities of global navigation satellite systems, status, and potential mitigation techniques. Proc IEEE 104(6):1174-1194. https://doi.org/10.1109/JPROC.2016.2535898

Ivanov AV, Komrakov D, Boykov DV (2015) Autonomous system for monitoring the integrity of navigation data provided by satellite navigation systems based on optimal information processing algorithms for navigation systems of land moving objects. In: Young Scientist USA, Vol. 4. Raleigh: Lulu. p. 71-78.

Kim D, Cho J (2018) Improvement of anomalous behavior detection of GNSS signal based on TDNN for augmentation systems. Sensors 18(11):3800. https://doi.org/10.3390/s18113800

Kruschke JK, Liddell TM (2018) Bayesian data analysis for newcomers. Psychon Bull Rev 25:155-177.

Layh T, Gebre-Egziabher D (2017) Design for graceful degradation and recovery from GNSS interruptions. IEEE Aerosp Electron Syst Mag 32(9):4-17. https://doi.org/10.1109/MAES.2017.160194

Mazumder S (2016) Numerical methods for partial differential equations: Finite difference and finite. Cambridge: Academic Press.

Onrubia R, Pascual D, Park H, Camps A, Rüdiger C, Walker JP, Monerris A (2019) Satellite cross-talk impact analysis in airborne interferometric global navigation satellite system-reflectometry with the microwave interferometric reflectometer. Remote Sens 11(9):1120. https://doi.org/10.3390/rs11091120

Pan L, Zhang X, Liu J, Li X, Li X (2017) Performance evaluation of single-frequency precise point positioning with GPS, GLONASS, BeiDou and Galileo. J Navig 70(3):465-482. https://doi.org/10.1017/S0373463316000771

Rufa JR, Atkins EM (2016) Unmanned aircraft system navigation in the urban environment: A systems analysis. J Aerosp Inf Syst 13(4):143-160. https://doi.org/10.2514/1.1010280 
Swamy KCT (2017) Global navigation satellite system and augmentation. Reson 22:1155-1174. https://doi.org/10.1007/ s12045-017-0579-6

Tran NQH, Prodan I, Grøtli EI, Lefèvre L (2018) Potential-field constructions in an MPC framework: Application for safe navigation in a variable coastal environment. IFAC-PapersOnLine 51(20):307-312.https://doi.org/10.1016/j.ifacol.2018.11.049

Van den Bergh B, Pollin S (2019) Keeping UAVs under control during GPS jamming. IEEE Syst J 13(2):2010-2021. https:// doi.org/10.1109/JSYST.2018.2882769

Venkatesh GA, Sumanth P, Jansi KR (2017) Fully autonomous UAV Proceedings. Paper presented 2017 International Conference on Technical Advancements in Computers and Communications (ICTACC). IEEE, Melmaurvathur, India. https://doi.org/10.1109/ICTACC.2017.20

Wang E, Jia C, Tong G, Qu P, Lan X, Pang T (2018) Fault detection and isolation in GPS receiver autonomous integrity monitoring based on chaos particle swarm optimization-particle filter algorithm. Adv Space Res 61(5):1260-1272. https:// doi.org/10.1016/j.asr.2017.12.016

Yang L, Zhang Y, Gao Y (2013) Enhanced RAIM based on weighted and subset schemes for GNSS receiver. Paper presented Proceedings of the ION 2013 Pacific PNT Meeting. ION; Honolulu, Hawaii, United States. [accessed Nov. 9 2021]. https:// www.ion.org/publications/abstract.cfm?articleID $=11028$ 